
associated with mid-latitude cyclones. J. Operational Meteor., 1 (7), 71-83, doi: http://dx.doi.org/10.15191/ nwajom.2013.0107.

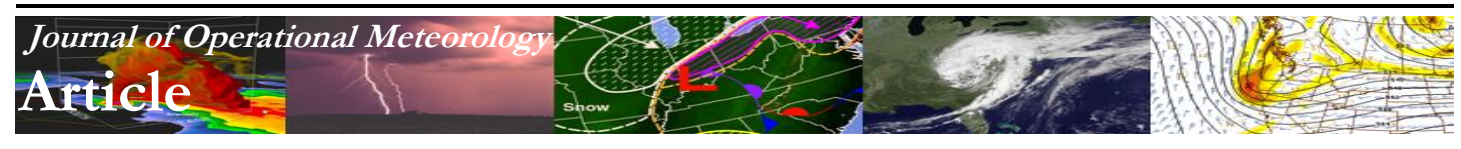

\title{
Multispectral Imagery for Detecting Stratospheric Air Intrusions Associated with Mid-Latitude Cyclones
}

\author{
BRADLEY T. ZAVODSKY and ANDREW L. MOLTHAN \\ NASA/Marshall Space Flight Center, Huntsville, Alabama \\ MICHAEL J. FOLMER \\ University of Maryland/ESSIC/CICS, College Park, Maryland \\ (Manuscript received 20 December 2012; in final form 18 March 2013)
}

\begin{abstract}
Introduction of upper-level potential vorticity (PV) to developing surface low pressure systems is a key component to cyclogenesis. These anomalous PV features generally occur in regions where stratospheric air has penetrated into the troposphere. Operational forecasters need tools to quickly identify PV anomalies via both cloud patterns and atmospheric characteristics because time is limited to make decisions and issue forecasts. A multispectral red, green, and blue (RGB) imagery product from the Moderate Resolution Imaging Spectroradiometer (MODIS) is presented that combines the utility of single-channel cloud information from infrared and water vapor imagery with information about ozone content into a single satellite image that assigns specific colors to specific atmospheric characteristics. Referred to as the Air Mass product, it provides detection of cloud and PV features associated with cyclogenesis as demonstrated for a Nor'easter in October 2011 by comparing the imagery to PV in Global Forecast System model analyses and total ozone from the Atmospheric Infrared Sounder. Development of the Air Mass RGB product from MODIS serves as a precursor and training tool for capabilities from the forthcoming operational Geostationary Operational Environmental Satellite R series Advanced Baseline Imager.
\end{abstract}

\section{Introduction}

Introduction of upper-level potential vorticity (PV) to surface disturbances is one well-documented factor driving cyclogenesis (e.g., Hoskins et al. 1985). Ertel's potential vorticity can be written (using standard meteorological assumptions) as:

$P V \equiv-g \eta_{\theta} \frac{\partial \theta}{\partial p}=$ constant

where $\eta_{\theta}$ is the absolute vorticity on an isentropic surface, $g$ is acceleration due to gravity, $\theta$ is the potential temperature, and $p$ is pressure. Anomalously large values of PV in the troposphere are commonly associated with stratospheric air intrusions (SAIs) that are introduced by tropopause folds (Uccellini 1990). An SAI results in a positive PV anomaly, which is associated with larger values of $\partial \theta / \partial p$ coincident with a positive absolute vorticity anomaly. When tropopause folds introduce enhanced PV in a region of low-level cyclogenesis, the cyclonic flow associated with the upper vortex enhances the surface anomaly resulting in deepening of the storm system (Danielsen 1968; Takayabu 1991). Total column ozone maxima are an appropriate proxy for SAI because descent of ozone-rich stratospheric air requires convergence in the lower stratosphere, which leads to local increases in the total column amount (Reed 1950). In fact, relationships have been found between absolute vorticity anomalies in the lower stratosphere and total column ozone (e.g., Vaughan and Price 1991; Olsen et al. 2000).

Fused data products allow operational forecasters to analyze more data and make decisions in a shorter time. Previous studies (e.g., Reader and Moore 1995; Bonasoni et al. 2000; Iacopelli and Knox 2001; Wimmers et al. 2003; Knox and Schmidt 2005) that have used satellite data to identify SAI have relied on multiple types of separate imagery and/or ozone sounding data. None of these projects have demonstrated a single satellite product for use by forecasters to quickly detect cloud features associated 
with developing extratropical cyclones and the SAI that may lead to rapid cyclogenesis. Multispectral composite red, green, and blue imagery (hereafter referred to as RGB) is a relatively new technique that could enhance forecaster decision-making abilities by reducing the number of datasets needed for analysis. Researchers at the European Organization for the Exploitation of Meteorological Satellites (EUMETSAT) have developed techniques based upon the Spinning Enhanced Visible and Infrared Imager (SEVIRI) aboard Meteosat-9 and -10 that combine ozone and airmass information. Through combinations of spectral bands focused on ozone concentration, temperature, and water vapor, the resulting product can be used to detect dry, ozone-rich stratospheric air associated with PV anomalies. These same techniques also can be applied to other satellite instruments that observe at similar wavelengths (EUMETSAT 2012a, b).

RGB imagery can be produced from the National Aeronautics and Space Administration (NASA) Moderate Resolution Imaging Spectroradiometer (MODIS) aboard the Terra and Aqua satellites and is used herein as a demonstration of capabilities that will be available from the National Oceanic and Atmospheric Administration (NOAA) Advanced Baseline Imager (ABI; Schmit et al. 2005), which will be aboard the Geostationary Operational Environmental Satellite R series (GOES-R) satellite currently slated for launch in late 2015. This geostationary satellite will provide a temporal frequency of around $15 \mathrm{~min}$ in full disk mode and 5 min in the continental United States sector that will enable forecasters to closely track storm development and its relationship to features such as PV anomalies associated with SAIs. Similar imagery also can be produced from the current GOES Sounder (e.g., Lindsey et al. 2011). The NASA Atmospheric Infrared Sounder (AIRS; Aumann et al. 2003), a hyperspectral infrared sounder collocated with MODIS aboard the Aqua satellite, is used herein as a complementary instrument to verify the presence of total-column ozone maxima associated with PV anomalies.

The objective of this paper is to demonstrate the utility of the Air Mass RGB imagery in detection of PV anomalies associated with SAI by analyzing the rapid deepening of an October 2011 Nor'easter that resulted in heavy snow, high winds, and expansive power outages across much of the northeastern United States. Sections 2 and 3 describe the RGB imagery and AIRS total ozone retrieval. Section 4 provides an overview of the October 2011 Nor'easter in terms of its PV anomaly characteristics, demonstration of total ozone in these PV anomaly regions, and application of the Air Mass RGB imagery in detection of both cloud and PV anomaly features.

\section{Multispectral composite imagery}

MODIS provides single-band imagery of albedo or brightness temperature over 36 spectral bands in the visible through infrared region of the spectrum allowing for remote sensing of land, ocean, and atmosphere characteristics. Selected visible and nearinfrared bands are sampled at $250-$ or $500-\mathrm{m}$ spatial resolution at nadir, while the remainder of the nearinfrared and infrared spectrum is sampled at $1 \mathrm{~km}$. Strategically combining a variety of wavelengths (or paired wavelength differences) and assigning red, green, and blue color intensities to each pixel allows for generation of a multispectral (i.e., false color) composite, or RGB, designed to characterize multiple features within a single image.

Herein, discussion will focus on application of the EUMETSAT Air Mass RGB product applied to the nearest SEVIRI-equivalent MODIS bands. The current EUMETSAT recipe for construction of the SEVIRI (MODIS) Air Mass RGB product assigns the 6.2-7.3 $\mu \mathrm{m}(6.75-7.33 \mu \mathrm{m})$ brightness temperature difference to the red intensity, the 9.7-10.8 $\mu \mathrm{m}(9.74-11.02 \mu \mathrm{m})$ brightness temperature difference to the green intensity, and the $6.2 \mu \mathrm{m}(6.75 \mu \mathrm{m})$ brightness temperature to the blue intensity. Red intensities correspond to temperature and moisture above any present cloud tops and differences in cloud emissivity at the 6.2 and $7.3 \mu \mathrm{m}$ wavelengths. Green intensities correspond to temperature differences between the surface $(10.8 \mu \mathrm{m})$ and any intervening cloud-free layer where there is significant ozone concentration (9.7 $\mu \mathrm{m})$, and surface emissivity differences among the 9.7 and $10.8 \mu \mathrm{m}$ channels in cloud free conditions. Since the $10.8 \mu \mathrm{m}$ channel will observe the temperature of the land surface under cloud free conditions, there is some diurnal and seasonal dependence upon contributions to the green color component. Finally, the blue color component corresponds to more traditional applications of single-band water vapor imagery. Where significant amounts of water vapor are present in the mid- to upper-troposphere, brightness temperatures cool and the blue contribution increases due to the inverted contribution of the blue channel. This is similar to grayscale water vapor 
imagery where colder brightness temperatures correspond to a brighter, whiter appearance.

Brightness temperatures or paired channel differences are converted to a single byte value (0-255) through application of a single equation that incorporates appropriate thresholds for each color intensity:

$$
(R, G, B)=255\left[\frac{(T B, \Delta T B)-M I N}{M A X-M I N}\right]^{\left(\frac{1}{\gamma_{R, G, B}}\right)}
$$

where (TB, $\Delta \mathrm{TB})$ means brightness temperature and brightness temperature difference, respectively, depending on the component. Byte values are calculated for the red, green, and blue values. Minimum and maximum temperature thresholds and the gamma correction parameter $\left(\gamma_{\mathrm{R}, \mathrm{G}, \mathrm{B}}\right)$ vary by product and input wavelengths. Their combination results in a 24-bit image with a vibrant color palette identifying a variety of features targeted by the product. Characteristics of the Air Mass RGB product are summarized in Table 1.

Table 1. Wavelengths and characteristics of inputs for the Air Mass RGB product developed by EUMETSAT for application to Meteosat-9 and -10/SEVIRI. $\gamma$ is the gamma correction parameter. Equivalent thresholds are applied to similar wavelength bands from MODIS. Note that the minimum and maximum thresholds for the blue channel result in an inversion of the color intensity as is typical for infrared imagery, where dark colors (black to dark blue) correspond to warm brightness temperatures and bright colors (vivid blue) correspond to cold brightness temperatures. Recipe from EUMETSAT (2012a).

\begin{tabular}{|c|c|c|c|c|}
\hline Color & Wavelengths & MIN & MAX & $\gamma$ \\
\hline $\mathrm{R}$ & $6.2-7.3 \mu \mathrm{m}$ & $-25 \mathrm{~K}$ & $0 \mathrm{~K}$ & 1.0 \\
\hline $\mathrm{G}$ & $9.7-10.8 \mu \mathrm{m}$ & $-40 \mathrm{~K}$ & $+5 \mathrm{~K}$ & 1.0 \\
\hline $\mathrm{B}$ & $6.2 \mu \mathrm{m}$ & $243 \mathrm{~K}$ & $208 \mathrm{~K}$ & 1.0 \\
\hline
\end{tabular}

Correct interpretation of the resulting RGB image is key to its successful application in an operational weather forecasting environment. Similar colors in the final RGB product correspond to synoptic or mesoscale features of interest to the operational forecaster. Figure 1 is the color interpretation guide for the Air Mass RGB image developed by EUMETSAT for SEVIRI data on Meteosat-9 and -10 that also applies to MODIS. Through use of the color key, combinations of multiple spectral bands in the false color Air Mass RGB product allow for quick interpretation of information that might otherwise be

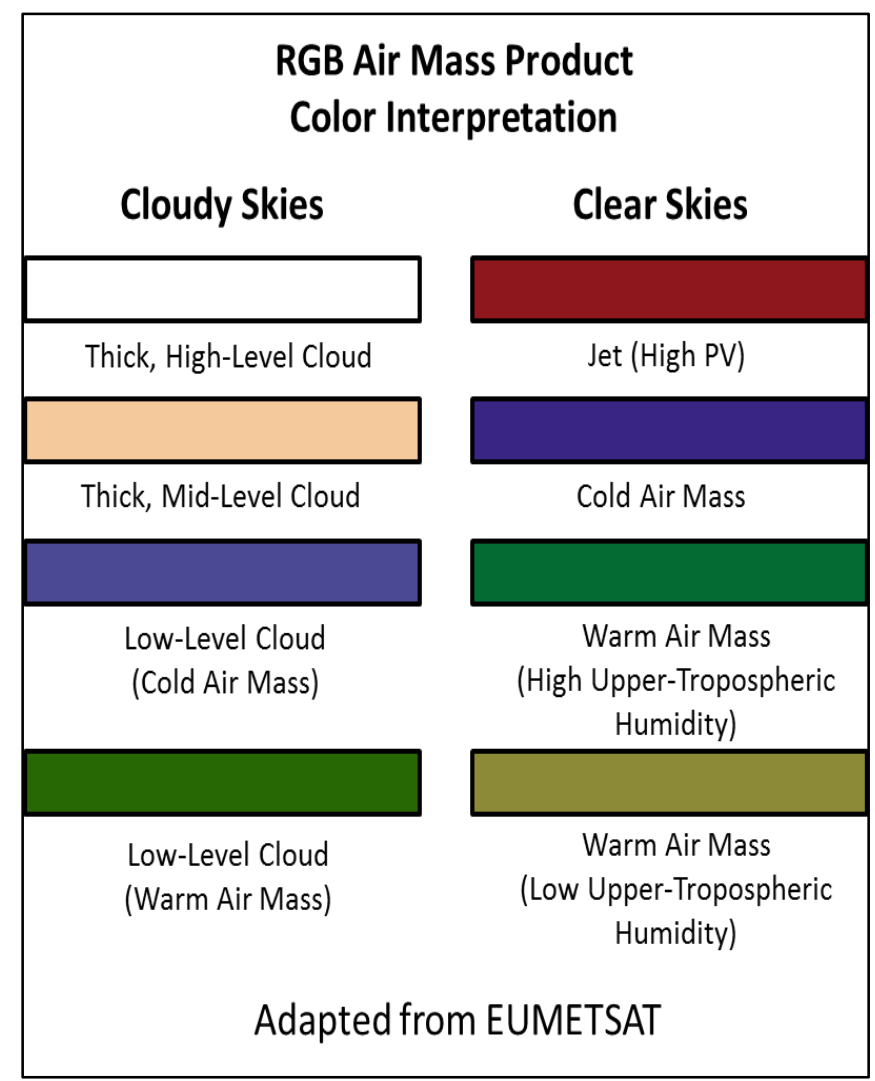

Figure 1. Color interpretation guide for the EUMETSAT Air Mass RGB product developed for Meteosat-9 and -10/SEVIRI as adapted from training materials provided by EUMETSAT (adapted from EUMETSAT 2012b). Additional training on interpretation of RGB products for SEVIRI is available at www.eumetrain.org. Click image for an external version; this applies to all figures hereafter.

understood only through time consuming evaluation of multiple single-channel images or products from multiple satellite instruments.

\section{AIRS total ozone product}

The AIRS and Advanced Microwave Sounding Unit (AMSU) form an integrated sounding system that can provide near-sonde quality atmospheric profiles in clear and partly cloudy fields of view. AIRS is a hyperspectral cross-track scanning spectrometer/ radiometer with 2378 spectral channels in the infrared and near-infrared. The enhanced spectral resolution of AIRS enables better vertical resolution and sounding accuracy over geostationary sounders that use only a few channels to derive the vertical column. The retrieved profiles have a horizontal spatial resolution of $45 \mathrm{~km}$ at nadir, but can be more than $100 \mathrm{~km}$ at the edge of scan. 


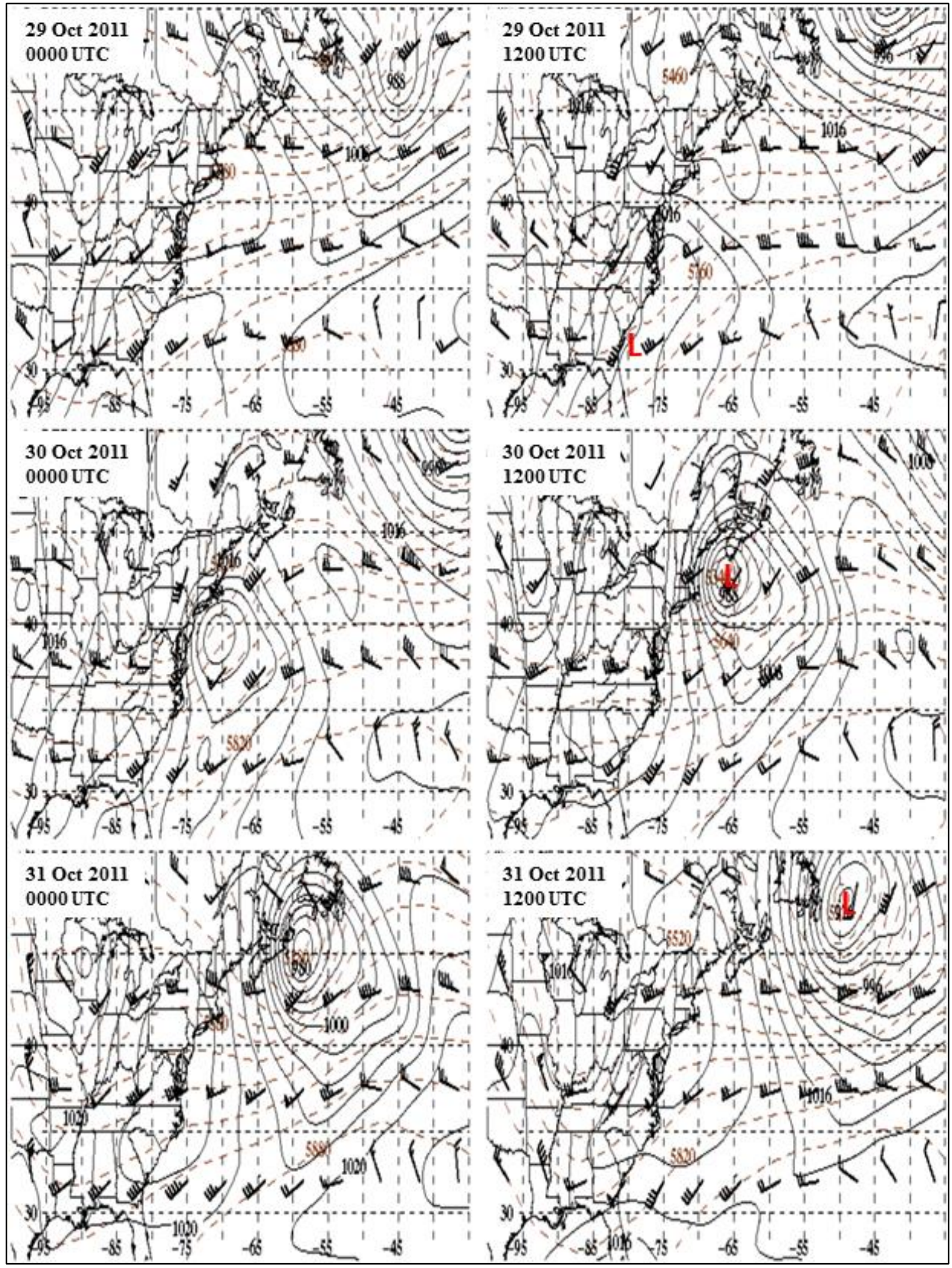

Figure 2. GFS Analysis from 29-31 October 2011 showing the development of a Nor'easter. Solid black contours are mean sea level pressure ( $\mathrm{hPa}$; contoured every $4 \mathrm{hPa}$ ), dashed brown contours are $500-\mathrm{hPa}$ geopotential height (m; contoured every $60 \mathrm{~m}$ ), and barbs are $300-\mathrm{hPa}$ winds $\left(\mathrm{m} \mathrm{s}^{-1}\right)$. The location of the surface low as analyzed by the NOAA Hydrometeorological Prediction Center is shown as a red "L" for the 1200 UTC plots. 
Version 5 (V5) Level 2 (L2) retrieved profiles from the operational version of the AIRS product retrieval software (originally described in Aumann et al. 2003) are used herein. The AIRS retrieval process is a multi-step method that includes an initial cloudclearing (Zhou et al. 2008) derived using observed radiances of a set of $3 \times 3$ array of AIRS fields of view and a physical retrieval (Susskind et al. 2003). In V5, the first guess for the ozone physical retrieval comes from an a priori ozone climatology (McPeters et al. 2007). Divakarla et al. (2008) found AIRS V5 total ozone retrievals contained a mean error of less than $3 \%$ compared to ozonesondes and global model analyses. Tian et al. (2007) and Pittman et al. (2009) have used AIRS retrieved profiles to determine areas of stratosphere-troposphere exchange.

A number of factors can reduce the quality of the AIRS retrieved profiles leading to a reduced spatial resolution from the nominal $50-\mathrm{km}$ spacing. Full retrievals in partly cloudy scenes can be obtained, but retrievals below thick clouds cannot; however, because the phenomenon being studied here is dry stratospheric air, necessary data regions are often cloud-free. To identify AIRS observations that are of the highest quality, a quality flag, Qual_O3 (Olsen et al. 2007), is employed. This Qual_O3 flag identifies ozone profiles in clear and partly cloudy conditions that are valid for weather studies. Here, only the highest quality total ozone retrievals (Qual_O3 = 0) are selected for inclusion in the AIRS total ozone analysis.

\section{Use of MODIS Air Mass RGB imagery to detect SAI}

\section{a. Case study overview: October 2011 Nor'easter}

An early season snowstorm affected much of the interior mid-Atlantic and northeastern United States from 29-30 October 2011 producing a rare white Halloween. Snowfall totals ranged from $35 \mathrm{~cm}$ (14 in) in West Virginia to $81 \mathrm{~cm}$ (32 in) in western Massachusetts. There were 39 deaths attributed to the storm with over 3.3 million people without power due to full-leaf trees falling on power lines. The storm also included wind gusts greater than $20 \mathrm{~m} \mathrm{~s}^{-1}$ (39 kt) along the mid-Atlantic states and greater than $25 \mathrm{~m} \mathrm{~s}^{-1}(50$ kt) from Nantucket to Cape Cod.

The storm underwent rapid cyclogenesis as it impacted the eastern United States with a central pressure drop from around $1012 \mathrm{hPa}$ at 0000 UTC 29 October 2011 to below $976 \mathrm{hPa}$ by 1200 UTC 31 October 2011. Figure 2 shows the Global Forecast

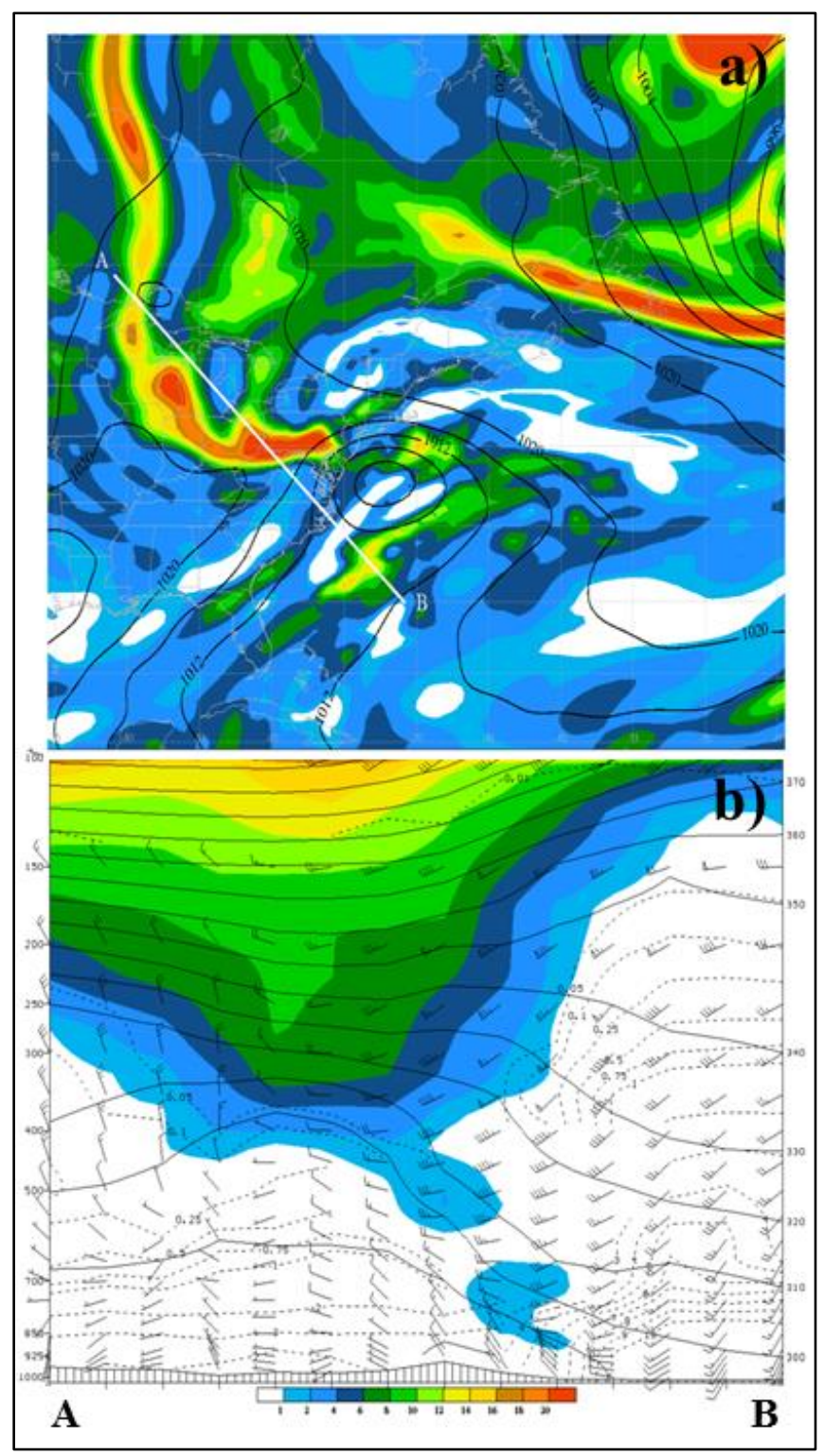

Figure 3. GFS analysis valid 1800 UTC 29 October 2011 for a) mean sea level pressure (solid black contours) and 400-500-hPa potential vorticity (colored contours; PVU $=10^{-6} \mathrm{~K} \mathrm{~m}^{2} \mathrm{~kg}^{-1} \mathrm{~s}^{-1}$ ) and b) vertical cross-section (extent represented by white line in "a") showing potential vorticity (colored contours; PVU), potential temperature (solid lines; $\mathrm{K}$ ), mixing ratio (dashed lines; $\mathrm{g} \mathrm{kg}^{-1}$ ), and winds (barbs; $\mathrm{m} \mathrm{s}^{-1}$ ).

System (GFS) model analyses from 29-31 October 2011 depicting the surface and upper-level setup that resulted in the Nor' easter. Figures 3a, 4a, and 5a show the 400-500-hPa layer PV (on pressure coordinates) for the GFS analysis times that most closely match the overpass times of the Aqua satellite on 29 and 30 October 2011. These levels were selected to match the analysis performed in Reader and Moore (1995). Figures $3 \mathrm{~b}, 4 \mathrm{~b}$, and $5 \mathrm{~b}$ show a cross section through 


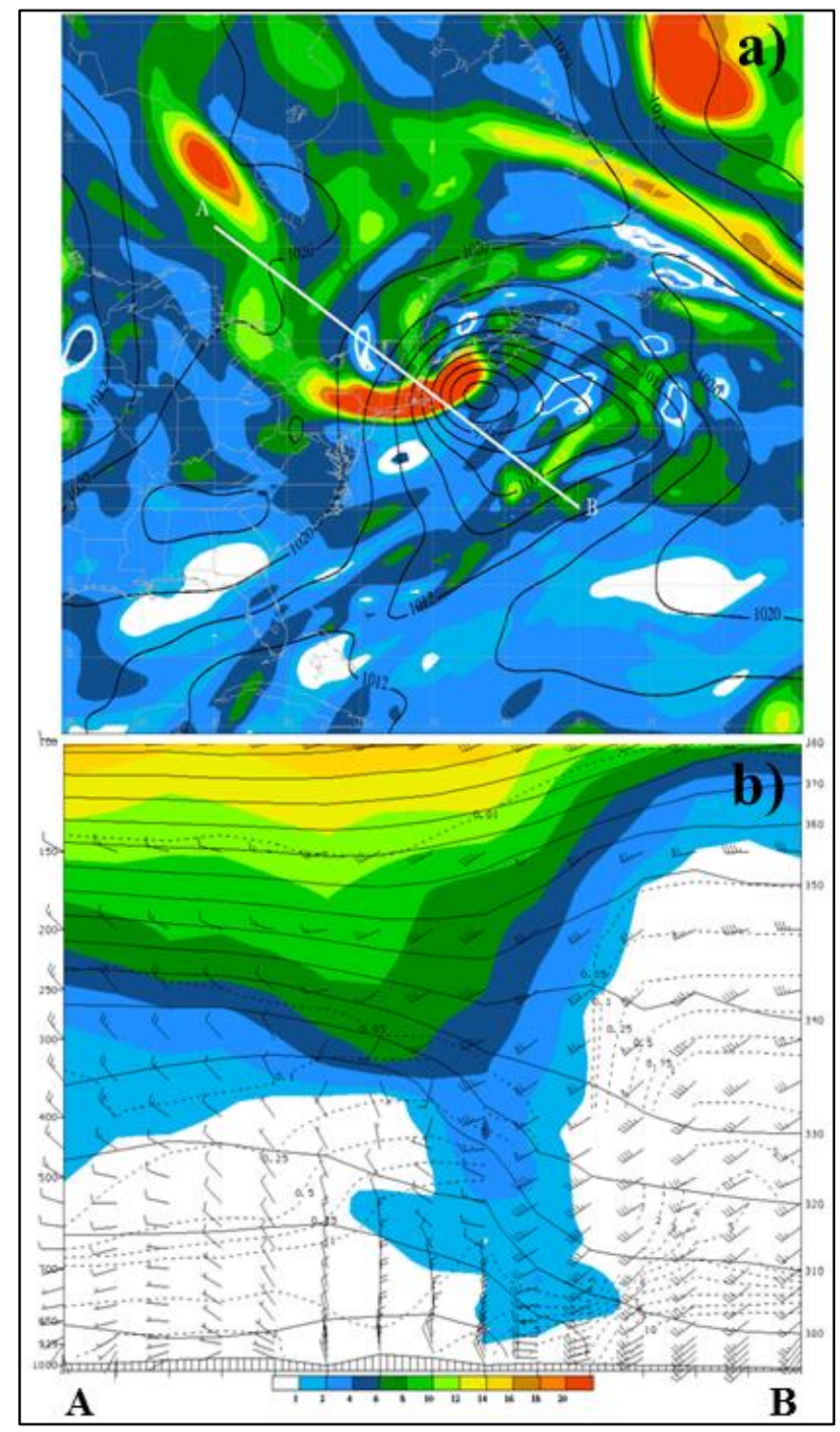

Figure 4. As in Fig. 3 but GFS analysis valid at 0900 UTC 30 October 2011. The white line in a) represents the cross section depicted in b).

the region of the PV anomaly on the western side of the surface low, where the upper-level vortex provided additional cyclonic circulation to the surface low. In the cross-section figures, the $2 \mathrm{PV}$ units (PVU; $1 \mathrm{PVU}$ $=10^{-6} \mathrm{~K} \mathrm{~m}^{2} \mathrm{~kg}^{-1} \mathrm{~s}^{-1}$ ) contour has been shown to be an appropriate definition of the tropopause (Thorncroft et al. 1993).

At 0000 UTC 29 October, a dip in the jet stream appeared over the upper Midwest as seen in the upperlevel winds and 500-hPa height fields. By 1800 UTC 29 October (Fig. 3a), the PV maximum associated with the upper-level vortex extended from southern Wisconsin through the Ohio Valley and into southern Pennsylvania. There was a residual PV maximum over the North Atlantic associated with a strong midlatitude cyclone that had propagated northward in the open waters of the Atlantic a few days prior, but this feature had no impact on the Nor'easter. The cross section from the 1800 UTC analysis (Fig. 3b) indicated elevated PVU values were as low as $600 \mathrm{hPa}$ over West Virginia (in the core of the PV anomaly) with the $2 \mathrm{PVU}$ contour at around $400 \mathrm{hPa}$, indicating the beginning of SAI into the troposphere. Additionally, in this region, the isentropic surfaces spread in the vertical and the mixing ratio was very low (e.g., $0.05 \mathrm{~g} \mathrm{~kg}^{-1}$ as low as $700 \mathrm{hPa}$ ), both indications of a tropopause fold associated with very dry stratospheric air. At 1800 UTC, the surface low, which was previously off the coast of the Carolinas (Fig. 2), had moved far enough north to begin interacting through convection with the upper-level trough and associated PV anomaly (Fig. 3a). As the PV anomaly captured the surface low, rapid intensification of the storm occurred in the next $24 \mathrm{~h}$.

At 0000 UTC 30 October, the surface low had become closed off and was situated off the New Jersey coast with a central pressure of around $1000 \mathrm{hPa}$. At 0900 UTC 30 October (Fig. 4a), the 3-h GFS forecast initialized at 0600 UTC (a proxy for the GFS analysis at this time) showed continued interaction of the PV anomaly region with the surface low, which had dropped more than $12 \mathrm{hPa}$ over this time to below 988 $\mathrm{hPa}$. The surface low was located just off the coast of Cape Cod, Massachusetts, and the PV anomaly extended from the Great Lakes through central New York and Pennsylvania and into southeastern Maine. It was around this time that the storm had its greatest impact on the New England coast with strong wind gusts over $25 \mathrm{~m} \mathrm{~s}^{-1}$ (50 kt) reported from Nantucket to Cape Cod. These strong winds corresponded in both time and space with the strengthening SAI in the southwestern quadrant of the storm. The cross section through the PV anomaly feature (Fig. 4b) reveals elevated PVU values down to the surface with the 2 PVU contour now as deep into the lower troposphere as $600 \mathrm{hPa}$.

At 1800 UTC 30 October (Fig. 5), the PV anomaly associated with the upper-level vortex extended from Ontario through northern New York to Nova Scotia. The central pressure of the surface low had fallen to $980 \mathrm{hPa}$ and continued to interact with the region of enhanced PV. The cross section through the PV anomaly and center of the storm (Fig. 5b) shows values of PVU greater than 2 extending nearly to the surface, again indicating a very deep SAI into the 
troposphere providing additional circulation feedback to the still-deepening surface low. PVU approaching 6 is seen as low as $500 \mathrm{hPa}$, which indicates a stronger SAI than was shown in the rapid cyclogenesis case in Reader and Moore (1995).

The surface low and upper-level jet continued to move northeastward over the next $24 \mathrm{~h}$ and were located off the coast of Newfoundland with a central pressure below $976 \mathrm{hPa}$ on 31 October. The storm continued to strengthen over the next several days, eventually bottoming out around $965 \mathrm{hPa}$ in the North Atlantic. Winds in excess of $25 \mathrm{~m} \mathrm{~s}^{-1}(50 \mathrm{kt})$ were observed over the open ocean south of the Canadian Maritimes during this time.

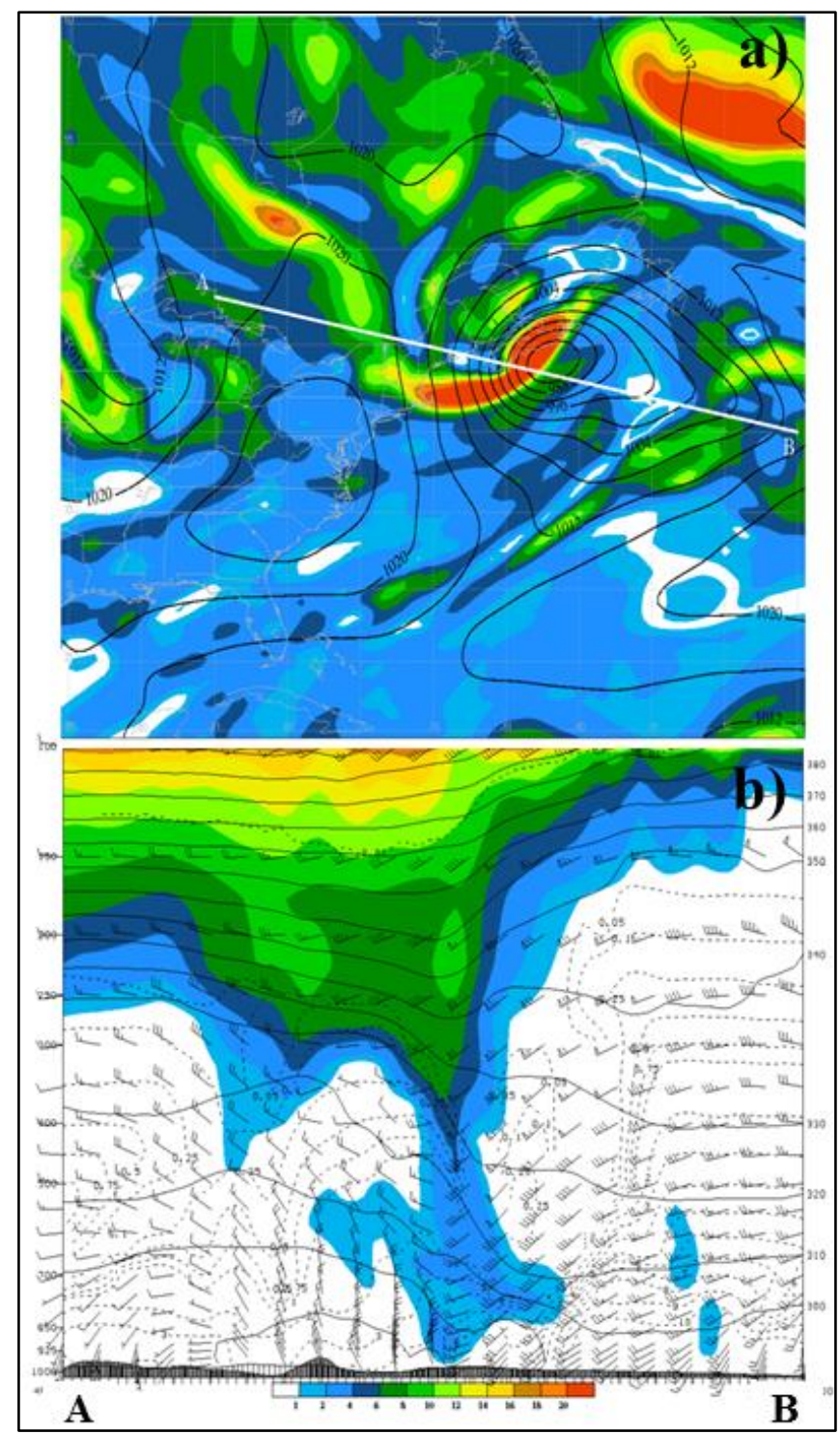

Figure 5. As in Figs. 3 and 4 but GFS analysis valid at 1800 UTC 30 October 2011. The white line in a) represents the cross section depicted in b).

\section{b. AIRS total ozone retrievals}

Total column ozone retrievals from AIRS are used here to provide further confirmation of SAI in high-PV regions. Figures 6-8 show AIRS L2 total column ozone profiles from the three overpasses of the Aqua satellite that occurred in the early stages of cyclogenesis on 29 and 30 October 2011. White spaces within the AIRS swath denote soundings that did not pass the quality control. These areas are generally associated with the deep convection and cloud cover from the storm system.

At 1800 UTC 29 October (Fig. 6), there were total ozone measurements that exceeded 450 Dobson Units (DU) in the clear area west of the storm over the Ohio Valley and West Virginia with a broad range of observations greater than 400 DU across much of the Great Lakes. This region of higher ozone concentration generally corresponded with the core of the upper-level trough with the high PV region in the GFS analysis to the south and west over southern Ohio. The PV anomaly region appeared along the gradient in the ozone values, where the absolute vorticity maximum was overlapped with large $\partial \theta / \partial p$ associated with the upper-level trough. The location of the SAI feature in the GFS cross-section compared favorably to the location of the highest total ozone concentration in the AIRS L2 profiles. The AIRS ozone values at 0900 UTC 30 October (Fig. 7) showed that the region of greater than 400 DU ozone shifted northeastward to upstate New York, following the upper-level vortex. Unfortunately, the edge of the AIRS swath bisected the anomalous PV region shown in the GFS analysis at this time; however, the high-PV area is qualitatively coincident with the southern edge of the gradient in total ozone observations from AIRS. Similarly, AIRS total ozone values at 1800 UTC 30 October (Fig. 8) showed greater than 400 DU (with embedded values exceeding $450 \mathrm{DU}$ ) again with the southern edge of the ozone gradient corresponding with the high-PV region in the GFS analysis, this time over Maine.

\section{c. MODIS Air Mass RGB}

Based on the recipe for generation of the Air Mass RGB image, the combination of high ozone concentration (strongly absorbed at $9.7 \mu \mathrm{m}$ resulting in a small contribution from green) and low water vapor content (resulting in a small contribution from blue) associated with SAI regions means regions of high PV should appear as a red coloration (see Fig. 1). Figures 


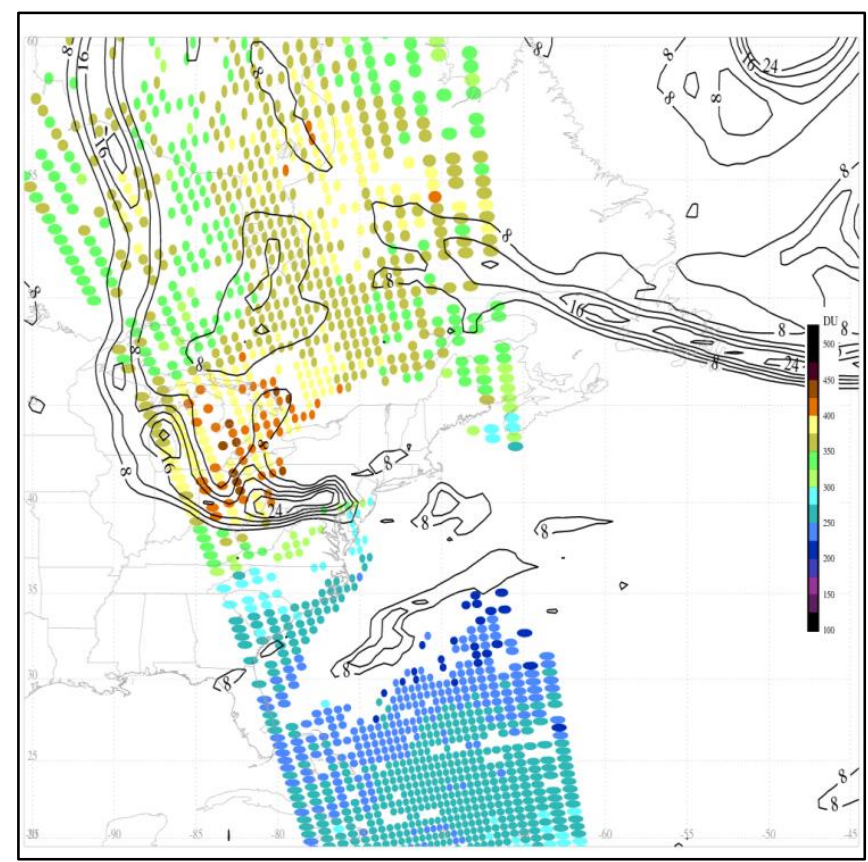

Figure 6. Total column ozone observations (Dobson Units) from AIRS at approximately 1800 UTC 29 November 2011. White areas within the AIRS swath represent profiles that did not pass quality control, which are generally associated with convective clouds. Black contours represent $400-500-\mathrm{hPa}$ potential vorticity (PVU; only contours greater than $8 \mathrm{PVU}$ are shown with a contour interval of 4 PVU) from the GDAS analysis valid at 1800 UTC 29 November 2011.

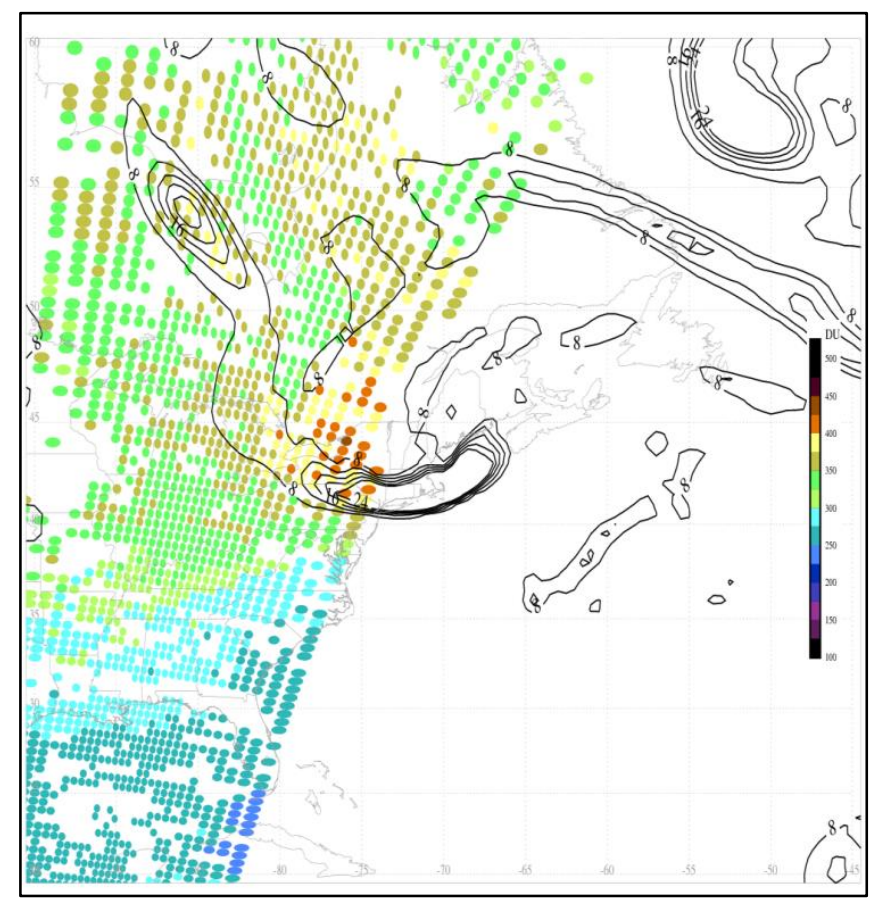

Figure 7. As in Fig. 6 but for AIRS profiles at approximately 0800 UTC 30 October 2011 and GDAS analysis valid at 0900 UTC 30 October 2011.

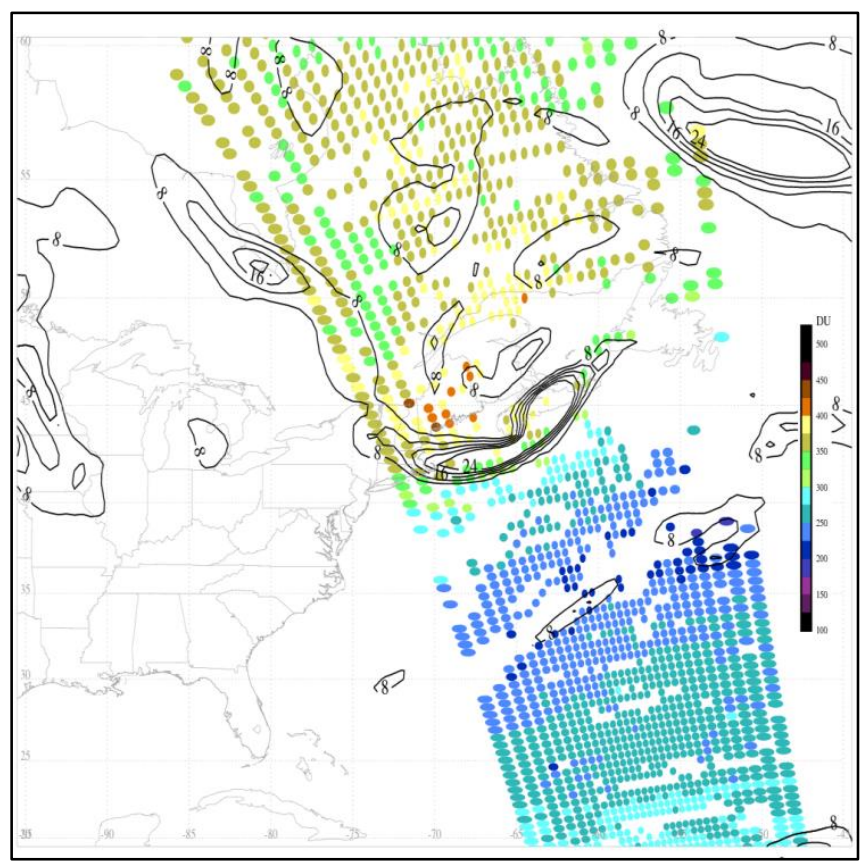

Figure 8. As in Figs. 6 and 7 but at approximately 1700 UTC 30 October 2011 and GDAS analysis valid at 1800 UTC 30 October 2011.

9-11 show the MODIS Air Mass RGB from the three overpasses of the Aqua satellite that occurred in the early stages of cyclogenesis on 29 and 30 October 2011. The MODIS Air Mass RGB at 1800 UTC 29 October (Fig. 9) detected a broad area of clouds in white extending from New England, westward to the Great Lakes, and southward along the Eastern Seaboard associated with the developing Nor'easter. The image also detected a broad area of red coloring from central Canada south through Wisconsin and Illinois and looping back through the Ohio Valley. This area was coincident with the area of SAI shown in the AIRS total column ozone (Fig. 6). The location of the PV anomaly in the GFS appeared along the southern edge of the red coloration, which was consistent with the relationship between the ozone gradient and the PV anomaly seen in Fig. 6. It should be noted that the MODIS RGB imagery at this time exhibited a known issue related to limb cooling, which led to cooler brightness temperatures in the water vapor channel along the swath edges. This reduction resulted in a slightly unrealistic darker red/purple coloration along the swath edges, which should not be considered when interpreting the imagery. At 0900 UTC 30 October, the Air Mass RGB image (Fig. 10) showed the red coloring across the Great Lakes, New York, Pennsylvania, New Jersey, and southern New England. Again, this location was qualitatively 


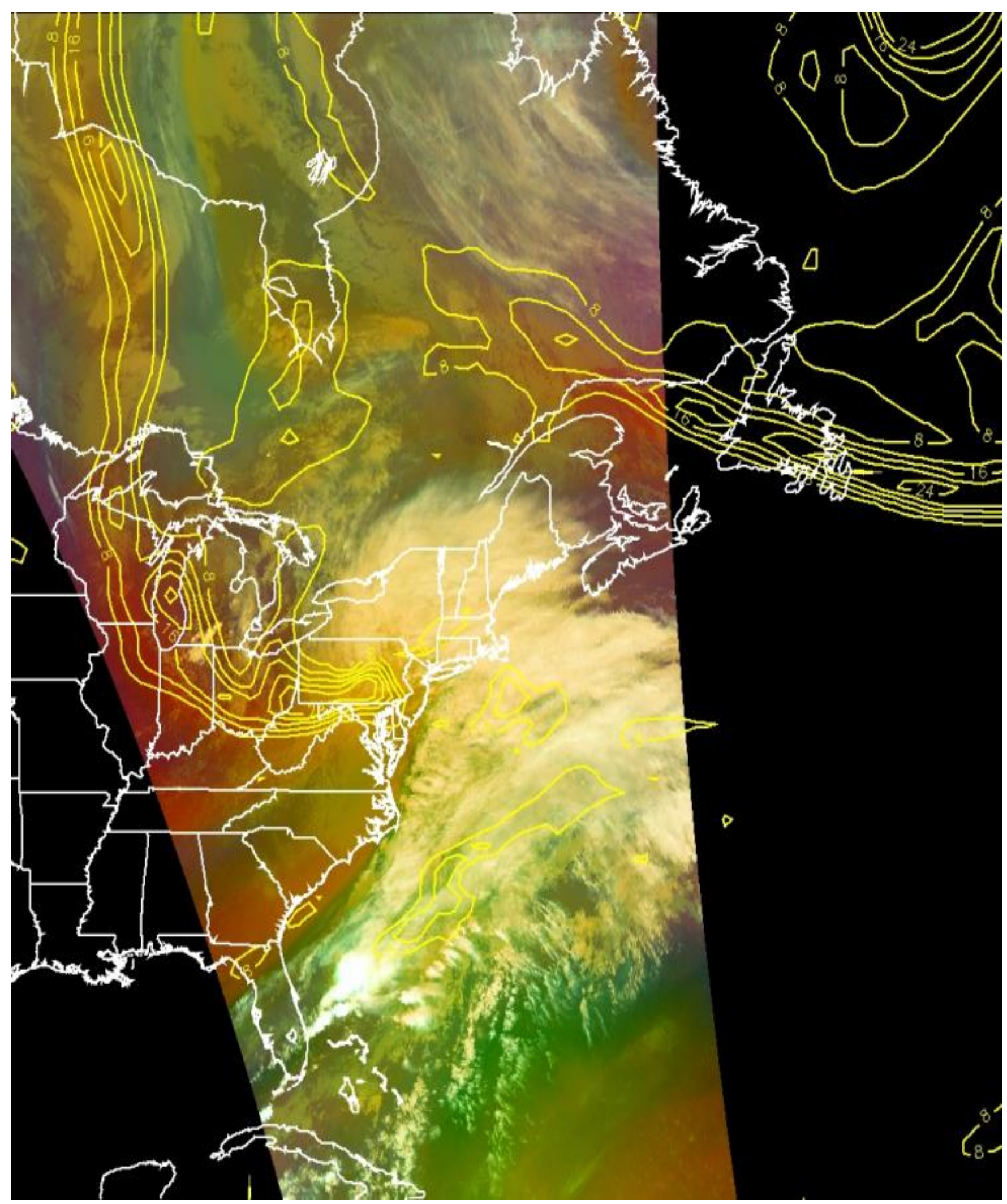

Figure 9. Aqua MODIS Air Mass RGB product at approximately 1800 UTC 29 October 2011. Yellow contours represent 400-500-hPa potential vorticity (PVU; only contours greater than 8 PVU are shown with a contour interval of 4 PVU) from the GDAS analysis valid at 1800 UTC 29 November 2011. 


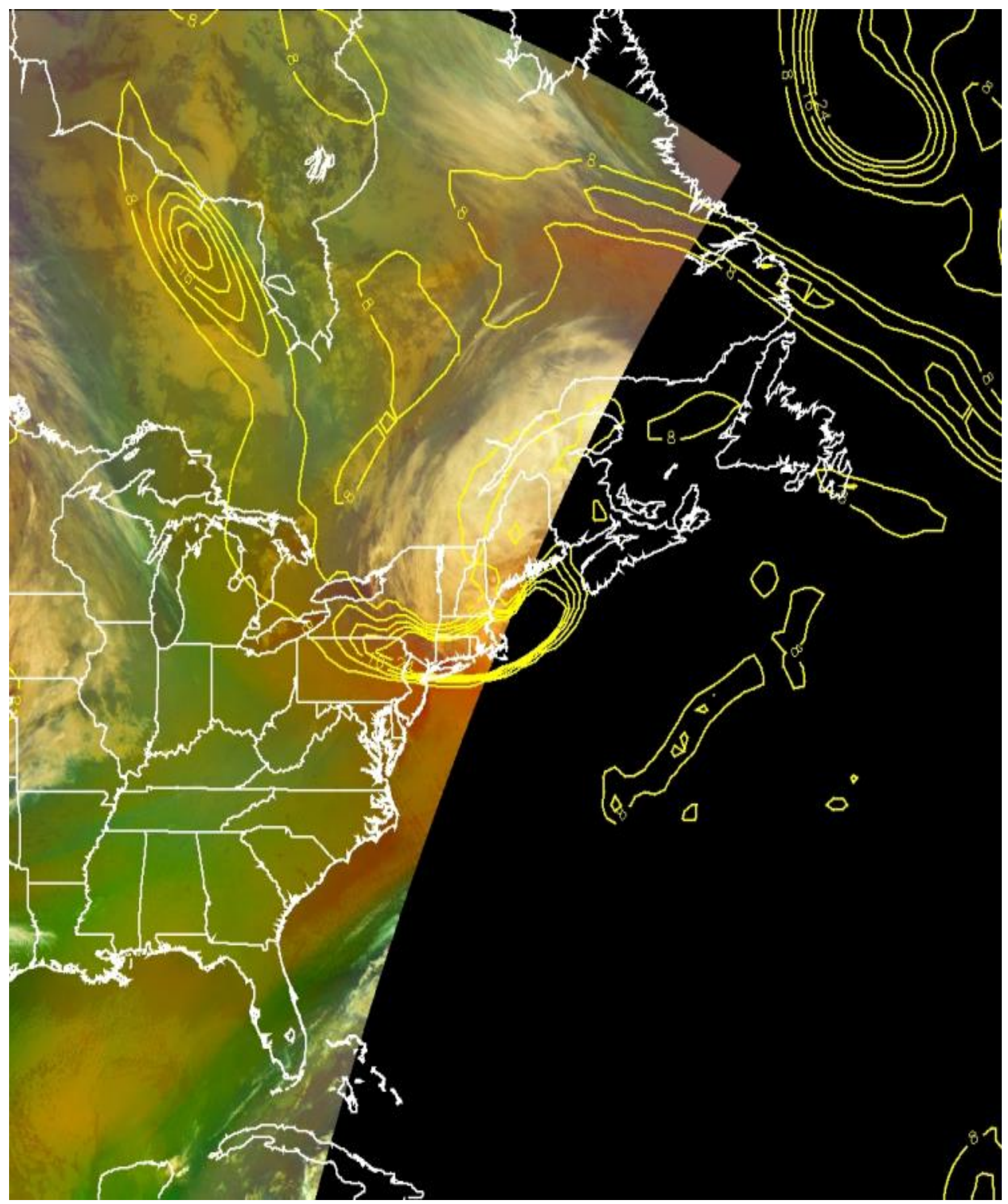

Figure 10. As in Fig. 9 but at approximately 0800 UTC 30 October 2011 and GDAS analysis valid at 0900 UTC 30 October 2011. 


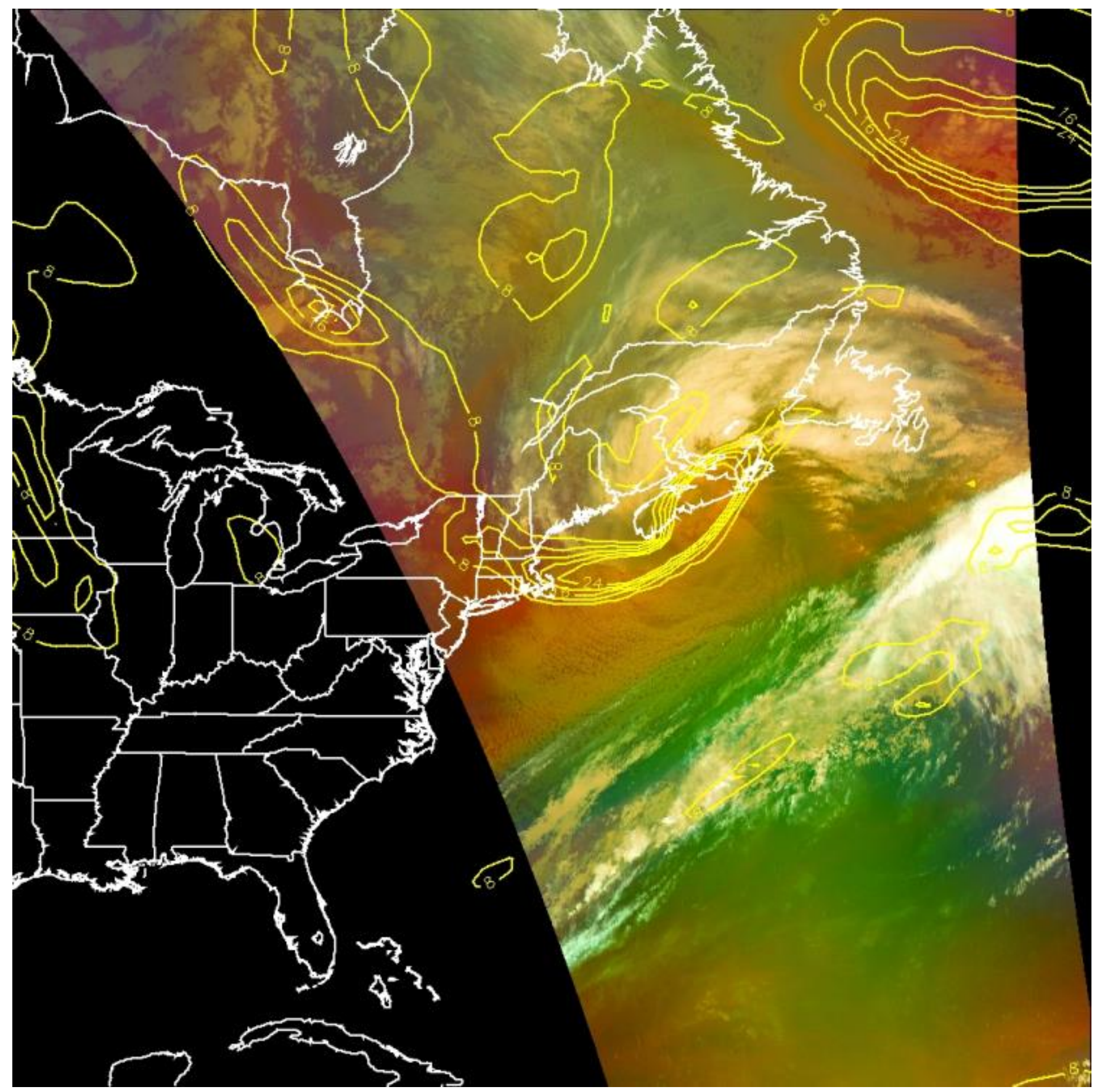

Figure 11. As in Figs. 9 and 10 but at approximately 1700 UTC 30 October 2011 and GDAS analysis valid at 1800 UTC 30 October 2011.

comparable to the location of SAI region in the AIRS ozone (Fig. 7). The southern edge of the most intense red coloration matched the location of the PV anomaly region from the GFS. The Air Mass RGB image at 1800 UTC 30 October (Fig. 11) again showed red coloration coincident with the area of total ozone concentration associated with the SAI. This region looped from southern Quebec through New York and
New England across part of the northern Atlantic and into Nova Scotia coincident with the advection of the PV around the western and southern edge of the storm as it continued to strengthen. The southern edge of the red coloration again matched the location of the PV anomaly.

The implication of the red coloration in the MODIS Air Mass RGB imagery matching the PV 
anomalies depicted in the GFS analysis and the elevated total ozone in the AIRS L2 retrievals is that forecasters can use the Air Mass RGB to determine whether stratospheric air and its associated PV is present in developing cyclones. While current evidence does not suggest that a darker shade of red coloration in the Air Mass RGB imagery has a qualitative correspondence to a deeper SAI or larger $\mathrm{PV}$ values, the development of the red features in the imagery over time can be tracked to determine how the mesoscale and synoptic scale environment of a system is changing to help forecasters fine tune the timing and placement of mid-latitude storm systems. The Air Mass RGB product has allowed forecasters to evaluate trends of important forcing mechanisms that drive shortwave evolution for cyclogenesis by determining movement of PV anomalies toward developing downstream features. The satellite trends from the Air Mass RGB imagery, when used along with the AIRS ozone and PV anomaly output from large-scale numerical analysis or model data, allow the forecaster to make a judgment on how well the model is performing, lending confidence to the overall forecast decision. While it may be challenging to track these changes using MODIS due to its temporal resolution, RGB imagery from GOES Sounder used in conjunction with MODIS allows forecasters to understand how to use RGB products from GOES-R with the temporal resolution of GOES and the spatial resolution of MODIS.

Currently, the relatively new Air Mass RGB product is used to confirm features seen in traditional satellite imagery and model analyses or forecasts. However, once forecasters have enough experience interpreting the RGB product, they can reduce the number of satellite products needed to identify and track relevant features associated with PV anomalies.

\section{Summary}

The ability to quickly identify both cloud patterns and multiple atmospheric characteristics is valuable in an operational forecasting environment where time is at a premium. A multispectral RGB imagery product has been presented that combines the utility of singlechannel cloud information from visible and water vapor imagery with information about ozone content into a single satellite image that forecasters can use for detecting PV anomalies associated with rapidly developing mid-latitude cyclones. SAI associated with $\mathrm{PV}$ anomalies in analysis products have been shown to be coincident with elevated total ozone concentrations from AIRS. Based on the recipe for generating the Air Mass RGB imagery, the expected red coloration associated with these areas has been confirmed for a rapidly developing Nor'easter from late October 2011. Forecasters can use the Air Mass RGB imagery to 1) see how the stratospheric air and PV are interacting with the cloud features of the storm and 2) provide observational evidence to gain confidence in numerical weather prediction (NWP) simulations that show rapid cyclogenesis.

The results presented here are directly applicable to Air Mass RGB imagery generated by multiple organizations from other platforms, such as the current GOES sounder, SEVIRI, and the forthcoming GOES$\mathrm{R}$ ABI. The use of the geostationary imagers for generation of the RGB products will provide forecasters with remotely sensed observations of SAI at greater temporal frequency than NWP models for supplemental synergy in tracking PV anomaly regions and their role in the evolution of cyclogenesis.

Acknowledgements. The authors thank Jochen Kerkmann of EUMETSAT for his collaboration in implementing the RGB recipe for MODIS. The authors also thank John Simko and Mark Ruminski of NOAA's Satellite Analysis Branch, Hugh McRandall of NOAA's Ocean Prediction Center, and Andrew Orrison of NOAA's Weather Prediction Center for their feedback on operational forecaster use and interpretation of the Air Mass RGB imagery. This work was supported by funding from the NASA Science Mission Directorate and NOAA GOES-R Proving Ground. MODIS data were acquired from NASA Level 1 and Atmosphere Archive and Distribution System (LAADS); AIRS data were acquired from the Goddard Earth Sciences Data and Information Services Center (GES DISC).

\section{REFERENCES}

Aumann, H. H., and Coauthors, 2003: AIRS/AMSU/HSB on the Aqua Mission: Design, science objectives, data products, and processing systems. IEEE Transactions on Geoscience and Remote Sensing, 41, 253-264.

Bonasoni, P., and Coauthors, 2000: Stratospheric ozone intrusion episodes recorded at Mt. Cimone during the VOTALP project: Case studies. Atmos. Environ., 34, 1355-1365.

Danielsen, E. F., 1968: Stratospheric-tropospheric exchange based on radioactivity, ozone, and potential vorticity. $J$. Atmos. Sci., 25, 502-518. 
Divakarla, M., and Coauthors, 2008: Evaluation of Atmospheric Infrared Sounder ozone profiles and total ozone retrievals with matched ozonesonde measurements, ECMWF ozone data, and Ozone Monitoring Instrument retrievals. J. Geophys. Res., 113, D15308, doi:10.1029/2007JD009317.

EUMETSAT, cited 2012a: Best practices for RGB compositing of multi-spectral imagery. [Available online at oiswww.eumetsat.int/ idds/html/doc/best_ practices.pdf.]

, cited 2012b: Airmass RGB. [Available online at oiswww.eumetsat.int/ idds/html/doc/airmass_interpreta tion.pdf.]

Hoskins, B. J., M. E. McIntyre, and A. W. Robertson, 1985: On the use and significance of isentropic potential vorticity maps. Quart. J. Roy. Meteor. Soc., 111, 877-946.

Iacopelli, A. J., and J. A. Knox, 2001: Mesoscale dynamics of the record-breaking 10 November 1998 mid-latitude cyclone: A satellite-based case study. Natl. Wea. Dig., $25(1,2), 33-42$.

Knox, J. A., and C. C. Schmidt, 2005: Using GOES total column ozone to diagnose stratospheric intrusions and nowcast non-convective cyclone windstorms: Methodology and initial results. Preprints, 13th Symp. on Meteorological Observations and Instrumentation, Savannah, GA, Amer. Meteor. Soc., 5.2. [Available online at ams.confex.com/ams/15AppClimate/ webprogram/Paper94099.html.]

Lindsey, D. T., and Coauthors, 2011: GOES-R Proving Ground Product Development at CIRA. National Weather Association 36th Annual Meeting, Birmingham, AL, Natl. Wea. Assoc., P4.17.

McPeters, R. D., G. J. Labow, and J. A. Logan, 2007: Ozone climatological profiles for satellite retrieval algorithms. J. Geophys. Res., 112, D05308, doi:10.1029/2005JD006823.

Olsen, E. T., and Coauthors, 2007: AIRS Version 5 Release Level 2 Standard Product QuickStart. Jet Propulsion Laboratory, Pasadena, CA. [Available online at: disc.sci.gsfc.nasa.gov/AIRS/documentation/v5 docs/AI RS_V5 Release User_Docs/V5_L2_Standard Product QuickStart.pdf].

Olsen, M. A., W. A. Gallus Jr., J. L. Stanford, and J. M. Brown, 2000: Fine-scale comparison of TOMS total ozone data with model analysis of an intense Midwestern cyclone. J. Geophys. Res., 105, D16, doi:10.1029/2000JD900205, 20487-20495.
Pittman, J. V., and Coauthors, 2009: Evaluation of AIRS, IASI, and OMI profile retrievals in the extratropical tropopause region using in situ aircraft measurements. J. Geophys. Res., 114, D24109, doi: 10.1029/2009JD012493.

Reader, M. C., and G. W. K. Moore, 1995: Stratospheretroposphere interactions associated with a case of explosive cyclogenesis in the Labrador Sea. Tellus, 47A, 849-863.

Reed, R. J., 1950: The role of vertical motions in ozoneweather relationships. J. Meteor., 7, 263-267.

Schmit, T. J., M. M. Gunshor, W. P. Menzel, J. J. Gurka, J. Li, and A. S. Bachmeier, 2005: Introducing the nextgeneration Advanced Baseline Imager on GOES-R. Bull. Amer. Met. Soc., 86, 1079-1096.

Susskind, J., C. D. Barnet, and J. Blaisdell, 2003: Retrieval of atmospheric and surface parameters from AIRS/AMSU/HSB data under cloudy conditions. IEEE Trans. Geosci. Remote Sens., 41 (2), 390-409, doi:10.1109/TGRS.2002.808236.

Takayabu, I., 1991: Coupling development. An efficient mechanism for the development of extratropical cyclones, J. Meteor. Soc. Japan, 69, 609-628.

Thorncroft, C. D., B. J. Hoskins, and M. E. McIntyre, 1993: Two paradigms of baroclinic-wave life-cycle behavior. Quart. J. Roy. Meteor. Soc., 119, 17-55.

Tian, B., Y. L. Yung, D. E. Waliser, T. Tyranowski, L. Kuai, E. J. Fetzer, and F. W. Irion, 2007: Intraseasonal variations of the tropical total ozone and their connection to the Madden-Julian Oscillation. Geophys. Res. Letters, 34, L08704, doi:10.1029/2007GL029451.

Uccellini, L. W., 1990: Processes contributing to the rapid development of extratropical cyclones. Extratropical Cyclones: The Erik Palmén Memorial Volume, C. Newton and E. H. Holopainen, Eds., Amer. Meteor. Soc., 81-105.

Vaughan, G., and J. D. Price, 1991: On the relation between total ozone and meteorology. Quart. J. Roy. Meteor. Soc., 117, 1281-1298.

Wimmers, A. J., and Coauthors, 2003: Signatures of tropopause folding in satellite imagery. J. Geophys. Res., 108, 8360, doi:10.1029/2001JD001358, D4.

Zhou, L., and Coauthors, 2008: Regression of surface spectral emissivity from hyper spectral instruments. IEEE Trans. Geosci. Remote Sens., 46 (2), 328-333, doi:10/1109/TGRS.2007.912712. 